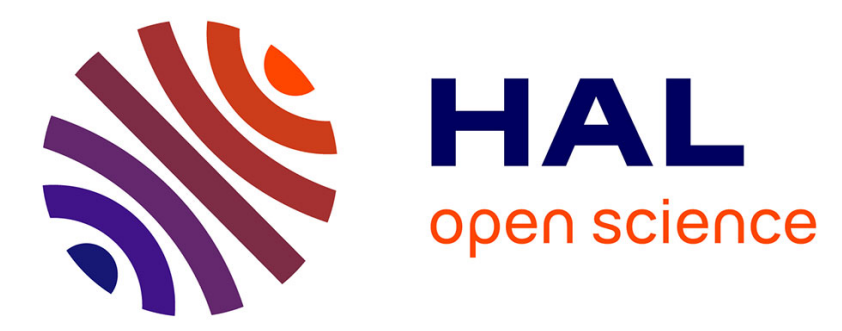

\title{
Investigation of Group Birefringence of Polarisation Maintaining Highly Birefringent Optical Fiber
}

Norbert Tarjanyi, Daniel Kacik, Raphael Jamier, Georges Humbert, Philippe Roy, Jean-Louis Auguste

\section{- To cite this version:}

Norbert Tarjanyi, Daniel Kacik, Raphael Jamier, Georges Humbert, Philippe Roy, et al.. Investigation of Group Birefringence of Polarisation Maintaining Highly Birefringent Optical Fiber. 2020 ELEKTRO, May 2020, Taormina, Italy. pp.1-4, 10.1109/ELEKTRO49696.2020.9130200 . hal-03436585

\section{HAL Id: hal-03436585 https://hal.science/hal-03436585}

Submitted on 19 Nov 2021

HAL is a multi-disciplinary open access archive for the deposit and dissemination of scientific research documents, whether they are published or not. The documents may come from teaching and research institutions in France or abroad, or from public or private research centers.
L'archive ouverte pluridisciplinaire HAL, est destinée au dépôt et à la diffusion de documents scientifiques de niveau recherche, publiés ou non, émanant des établissements d'enseignement et de recherche français ou étrangers, des laboratoires publics ou privés. 


\section{Investigation of Group Birefringence of Polarisation Maintaining Highly Birefringent Optical Fiber}

\author{
Norbert Tarjányi \\ Department of Physics \\ Faculty of Electrical Engineering and \\ Information Technology, University of \\ Žilina \\ Žilina, Slovakia \\ tarjanyi@fyzika.uniza.sk \\ Georges Humbert \\ University of Limoges, CNRS \\ XLIM, UMR 7252 \\ 87000 Limoges, FRANCE \\ Limoges, France \\ georges.humbert@xlim.fr
}

\author{
Daniel Káčik \\ Department of Physics \\ Faculty of Electrical Engineering and \\ Information Technology, University of \\ Žilina \\ Žilina, Slovakia \\ kacik@fel.uniza.sk \\ Philippe Roy \\ University of Limoges, CNRS \\ XLIM, UMR 7252 \\ 87000 Limoges, FRANCE \\ Limoges, France \\ philippe.roy@xlim.fr
}

\author{
Raphael Jamier \\ University of Limoges, CNRS \\ XLIM, UMR 7252 \\ 87000 Limoges, FRANCE \\ raphael.jamier@xlim.fr \\ Jean-Louis Auguste \\ University of Limoges, CNRS \\ XLIM, UMR 7252 \\ 87000 Limoges, FRANCE \\ jean-louis.auguste@xlim.fr
}

\begin{abstract}
In the paper there are presented results of an investigation of a group birefringence of a fabricated polarization maintaining highly birefringent optical fiber around the central wavelength $1550 \mathrm{~nm}$. The value of group birefringence was determined in two experimental ways. The first experimental set-up uses a system based on fiber loop mirror and the second one is based on a standard polariscopelike geometry usually used for investigation of birefringence of any optically transparent anisotropic medium. In both cases the birefringence is determined by appropriate analysis of typical interference patterns measured by the particular setup. The measurement was performed for fiber samples with various lengths. Comparison between values obtained for various lengths of samples and resulting from interference patterns measured using different experimental set-ups shows a very good match. Keywords-birefringence, Hi-Bi optical fiber, polarization, PM fiber, group delay
\end{abstract}

\section{INTRODUCTION}

Highly birefringent (Hi-Bi) optical fibers and $\mathrm{Hi}-\mathrm{Bi}$ microstructured, or Hi-Bi photonic crystal fibers are special optical fibers realized by introducing certain elements with special geometry and appropriate mechanical and optical properties ensuring the creation of a permanent and welldefined birefringence [1]. These kinds of fibers can be used as active parts of an optical fiber sensor of any physical quantity which significantly influences the fiber's birefringence [2-7]. In order to interpret the impact of the environment on the fiber's birefringence correctly, one has to know the dispersion of the birefringence of the fiber within the used spectral range, first. In the paper we present the result of investigation leading to determination of the group birefringence of the fabricated Hi-Bi fiber. The front face of the fiber is illuminated by a linearly polarized light from a SLED source with central wavelength $1550 \mathrm{~nm}$ and $100 \mathrm{~nm}$ spectral width and the end face of the fiber is connected to a fiber plane polarizer. The group birefringence dispersion is determined from the positions of subsequent maxima of the interference pattern measured by an optical spectrum analyzer. The value of the birefringence obtained is then compared to those gained by measurement using a system based on fiber loop mirror [8] and calculated from a differential group delay [9], as well.

\section{EXPERIMENTAL}

\section{A. Hi-Bi fiber fabrication and measurement of the group} birefringence using a fiber loop mirror

The experimental Hi-Bi fiber with cross-section shown in Fig. 1 was prepared at Xlim Research Institute. The outer diameter of the fiber is $140 \mu \mathrm{m}$, the diameter of the core equals $8.5 \mu \mathrm{m}$, the diameters of the two Stress Applied Parts (SAPs) located aside and symmetrically with respect to the fiber's core is $25 \mu \mathrm{m}$.

In the design of the polarisation maintaining (PM) fibre, the SAPs are composed of a silica-based glass called SAL $\left(\mathrm{SiO}_{2}-\mathrm{Al}_{2} \mathrm{O}_{3}-\mathrm{La}_{2} \mathrm{O}_{3}\right)$ with: $70 \%$ mol $\mathrm{SiO}_{2}+20 \% \mathrm{~mol} \mathrm{Al}_{2} \mathrm{O}_{3}+$ $10 \% \mathrm{~mol} \mathrm{La}_{2} \mathrm{O}_{3}$. At the opposite of a commercial Panda fibre, we introduced SAP where the refractive index of the material is larger than the refractive index of pure silica, with a strong attenuation coefficient. The preform is realised via the stack and draw technique and the core is coming from a drawing of a MCVD preform to obtain a germanium doped rod. The two capillaries are stacked to realise the SAP, and SAL glass is introduced in these capillaries via the powder in tube technique. The attenuation losses of the prepared fiber equal $0.06 \mathrm{~dB} / \mathrm{m}$ at $1550 \mathrm{~nm}$ for $\mathrm{LP}_{01}$ mode.

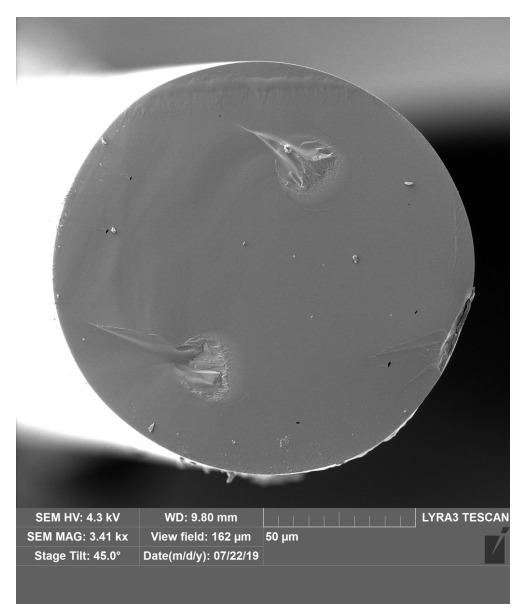

Fig. 1. SEM image of the front face of the fabricated PM Hi-Bi fiber. 


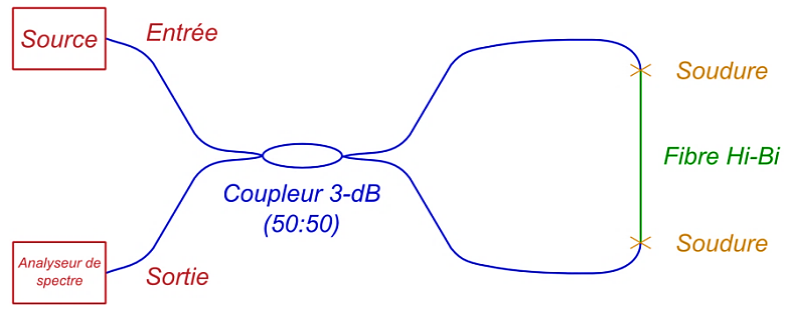

Fig. 2. FLM scheme with Hi-Bi fiber.

Birefringence of the fibre can be measured by the introduction of a PM fiber in a Sagnac Interferometer [10], or in an equivalent system based on a loop (Fiber Loop Mirrors, FLM) [11]. The birefringence measurement is done by measuring the interference of two contra-propagating waves guided in the same optical fibre, thus exposed to the same external perturbations [8]. FLM, as illustrated by Fig. 2, is composed of a 50/50 coupler, where the two ends are spliced to the Hi-Bi fiber. After analysing the interference pattern measured for fibers with the lengths ranging from approximately $11 \mathrm{~cm}$ to $115 \mathrm{~cm}$ the mean value of birefringence was determined to be $\Delta n=3.19 \cdot 10^{-4}$.

\section{B. Determinantion of the group birefringence using standard polariscope-like geometry}

The experimental setup for investigation of the group birefringence of the highly birefringent optical fiber is shown in Fig. 3. As a light source we used a linearly polarized source SLED (Safibra OFLS-6) with central wavelength $1550 \mathrm{~nm}$ and $100 \mathrm{~nm}$ FWHM. The signal was measured by optical spectrum analyzer (Anritsu MS9710B) with $0.07 \mathrm{~nm}$ resolution. For analyzing the polarization state of light emerging from the Hi-Bi fiber we used a plane polarizer $(\mathrm{OZ}$ Optics LTD.) integrated with a single mode (SM) fiber and designed for $1550 \mathrm{~nm}$ with possibility to change the polarization state in $360^{\circ}$.

The lengths of the two tested fiber samples were $138 \mathrm{~cm}$ and $50 \mathrm{~cm}$. The sample under the test was placed between two SM fibers and for alignment of the fibers we used the microstages NanoMax 300 (Thorlabs). Due to the sensitivity of the Hi-Bi fiber to the temperature changes, the fiber together with the microstages was placed in a temperature isolated chamber.

The investigation was performed at room temperature and normal atmospheric pressure. For given polarization state of the optical source we changed the position of the plane polarizer over the $360^{\circ}$ range with $5^{\circ}$ steps and observed the changes of the spectral distribution of intensity at the output of the SM fiber with integrated polarizer (Fig. 4). In figures $5 \mathrm{a}$ and $5 \mathrm{~b}$ there are plotted curves showing the typical intensity patterns, measured for a certain angle of rotation of the SM fiber integrated plane polarizer, as a function of wavelength for fiber samples $138 \mathrm{~cm}$ and $50 \mathrm{~cm}$ long, respectively.

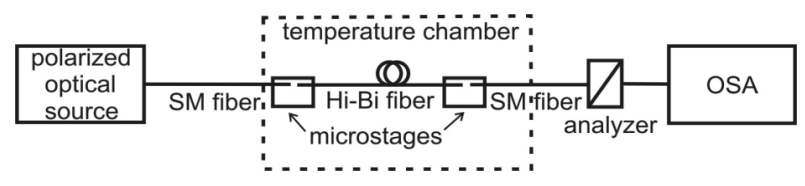

Fig. 3. Experimental set-up for investigating the group birefringence of the Hi-Bi fiber.

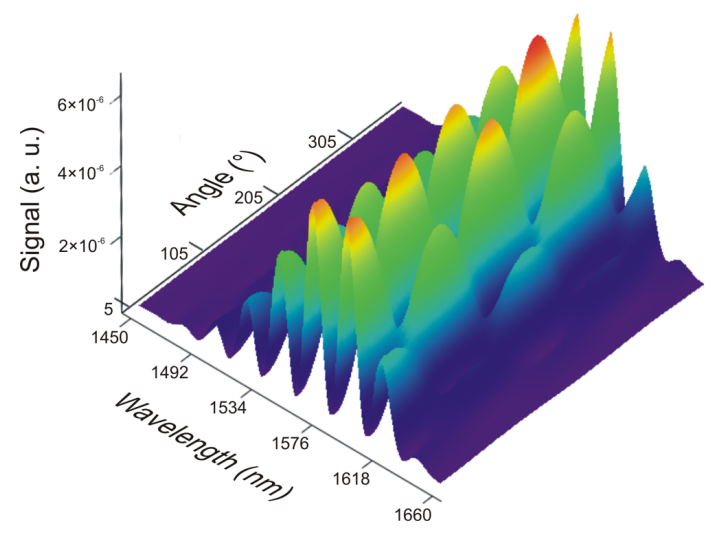

Fig. 4. Distribution of intensity measured at the output of the SM fiber with integrated polarizer as function of the polarizer's adjustment angle.

According to theory [12] such intensity, or interference pattern, is observed when a slightly birefringent medium is placed in between two plane polarizers with polarization planes rotated with respect to each other by an angle $\gamma$ and the medium is being illuminated by a broadband light source. In our case, however, the role of the first polarizer is directly fulfilled by a plane polarized broadband source of light.

In principle, there are many possible angles of rotation of the integrated plane polarizer for which the intensity distribution will look similarly like those shown in Fig. 5. Depending on the angle of rotation, however, the observed dependences will differ from each other by the magnitude and the contrast, i.e. by the difference in magnitude between consecutive minimum and maximum of the signal [13]. For our investigation such angle of rotation of the integrated plane polarizer was chosen for which the measured intensity pattern shows the maximal contrast.
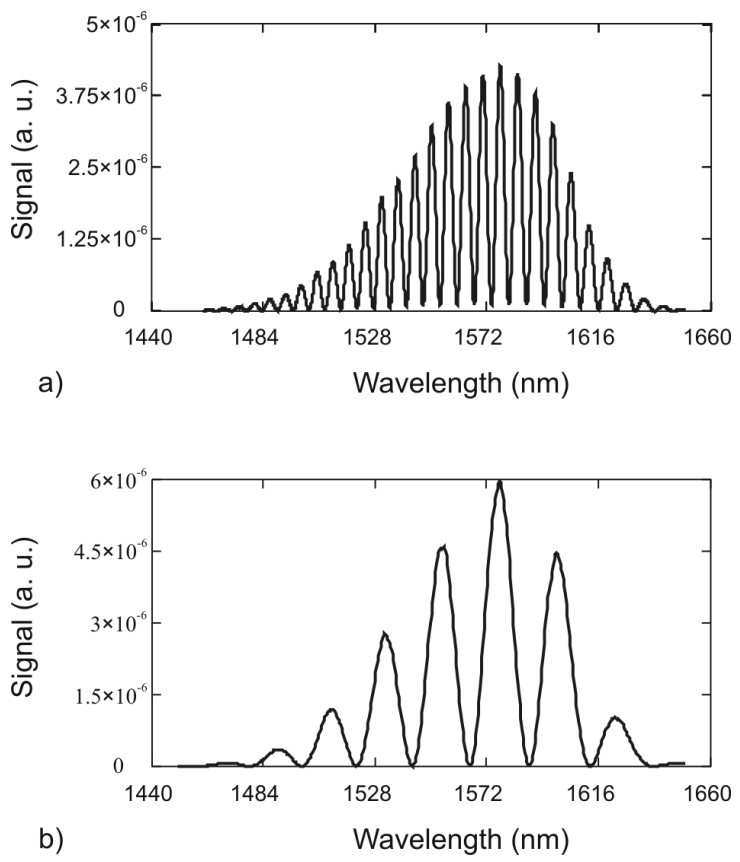

Fig. 5. Spectral distribution of intensity at the output of the plane polarizer for a chosen value of angle of rotation of the polarizer and length of the fiber sample $138 \mathrm{~cm} \mathrm{(a)} \mathrm{and} 50 \mathrm{~cm} \mathrm{(b).}$ 
From the measured intensity pattern and the known length $d$ of the birefringent fiber the group birefringence dispersion $\Delta n$ can be estimated by identifying the positions of subsequent minima and/or maxima, appearing due to interference of eigenmodes at the integrated optical polarizer, according to [13]

$$
\Delta n=\left(\lambda_{1} \cdot \lambda_{2}\right) /\left[d \cdot\left(\lambda_{1}-\lambda_{2}\right)\right],
$$

where $\lambda_{1}$ and $\lambda_{2}$ are the wavelengths corresponding to two subsequent maxima (minima). After determining the wavelengths corresponding to maxima and minima the group birefringence of the fibers under the test was computed according to Eq. (1). These values were then assigned to the wavelengths corresponding to central wavelengths of the particular intervals $\left(\lambda_{1}, \lambda_{2}\right)$ and plotted. The resulted values of birefringence obtained from interference patterns measured for lengths of the fiber samples $138 \mathrm{~cm}$ and $50 \mathrm{~cm}$ are shown in Fig. 6a and 6b, respectively.

From the measured interference pattern the group birefringence can be estimated also using the differential group delay approach [9]. By counting the number $N$ of extrema observed within the measured spectral span defined by wavelengths $\lambda_{b}$ and $\lambda_{e}$ representing the start and stop wavelengths, respectively and using relationship between differential group delay $\Delta \tau$ and the optical path difference, i.e. $\Delta \tau \cdot c=\Delta n \cdot d$, where $c$ is the speed of light in vacuum, one gets from the expression for the group delay of the fiber $\Delta \tau$ [9] the relationship for the group birefringence $\Delta n$

$$
\Delta n=\left(k \cdot N \cdot \lambda_{\mathrm{b}} \cdot \lambda_{\mathrm{e}}\right) /\left[2 d \cdot\left(\lambda_{\mathrm{b}}-\lambda_{\mathrm{e}}\right)\right],
$$

where parameter $k$ is a dimensionless mode-coupling factor statistically accounting for the wavelength dependence of the polarization states [9]. According to $\mathrm{Hi}-\mathrm{Bi}$ fiber cross section as shown in Fig. 1, we can assume that polarization modes emerging in the fiber and propagating through are coupled very weakly, if at all, resulting in $k=1$ as valid for nonmode-coupled fibers [9].

For number of extrema $N=57$ counted within the spectral range from $\lambda_{\mathrm{b}}=1460 \mathrm{~nm}$ to $\lambda_{\mathrm{e}}=1650 \mathrm{~nm}$ and length of the fiber sample $d=1.38 \mathrm{~m}$ we get from the Eq. (2) the value of $\Delta n=2.62 \cdot 10^{-4}$. Similarly, for number of extrema $N=17$ counted within the spectral range from $\lambda_{\mathrm{b}}=1485 \mathrm{~nm}$ to $\lambda_{\mathrm{e}}=1635 \mathrm{~nm}$ and length of the fiber sample $d=0.5 \mathrm{~m}$ we get from the Eq. (2) the value of $\Delta n=2.78 \cdot 10^{-4}$.

In both cases the range of wavelengths used for calculation of group birefringence was chosen so that the amplitude of the measured signal was sufficient for unambiguous distinguishing between a minimum and a maximum localized close to the starting and ending wavelengths of the measured spectrum. As it is shown in Fig. $6 \mathrm{a}$ and $6 \mathrm{~b}$, the value calculated according to Eq. (2) corresponds well with values obtained using Eq. (1) and can be considered a mean value within the investigated interval of wavelengths.
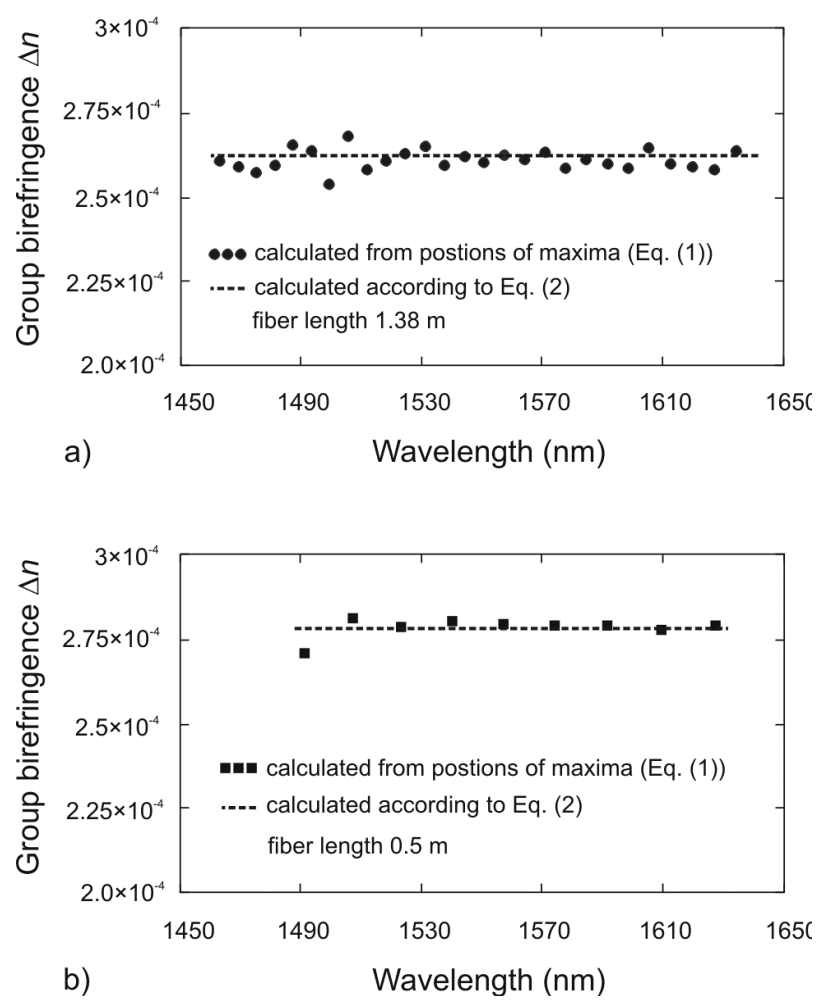

Fig. 6. Computed group birefringence of the experimental Hi-Bi optical fiber from interference pattern measured for fiber with length $138 \mathrm{~cm}$ (a) and $50 \mathrm{~cm}(\mathrm{~b})$.

\section{CONCLUSION}

The polarization maintaining highly birefringent optical fiber was designed and fabricated, and the group birefringence dispersion of the fiber in the spectral range from $1450 \mathrm{~nm}$ to $1650 \mathrm{~nm}$ was investigated. The group birefringence was determined first by analyzing the interference pattern observed when the fiber was a part of the loop fiber mirror. After that the plane polariscope-like set-up was used to determine the group birefringence using two approaches. First, the birefringence was calculated from known positions of maxima of the interference pattern and fiber's length $d$ according to Eq. (1) which was derived from condition of observing interference maxima (minima). Second, from the expression for the differential group delay of the fiber we derived expression for the group birefringence and calculated the mean value of $\Delta n$ using known number of extrema observed within the measured spectral range, length of the fiber and assuming the very weak coupling of the polarization eigenmodes. A good match between values obtained by these two approaches as well as the value obtained by FLM method was achieved.

\section{ACKNOWLEDGMENT}

This collaborative work was supported by Slovak Research and Development Agency under the projects no. SK-FR-2017-0017, PHC Stefanik 2018 NOFSEBII, APVV15-0441 and Slovak National Grant Agency under the projects no. VEGA 1/0540/18, VEGA 1/0069/19. 


\section{REFERENCES}

[1] M. Alam, D. Guertin, J. Farroni, J. Abramczyk, N. Jacobson, and K. Tankala, "Small form-factor PANDA type HiBi fiber for sensing applications," Proc. SPIE 5272, pp. 65-74, 2004.

[2] O. Frazão, D. Egypto, L. A. Bittencourt, M. T. M. R. Giraldi, and M. B. Marques, "Temperature sensor using Hi-Bi erbium-doped fiber loop mirror," Microwave and Optical Technology Letters, vol. 50, no. 12, pp. 3152-3154, 2008.

[3] S. Rota-Rodrigo, A. M. R. Pinto, M. Bravo, and M. Lopez-Amo, “An in-reflection strain sensing head based on Hi-Bi photonic crystal fiber," Sensors, vol. 13, pp. 8095-8102, 2013.

[4] P. Hlubina, T. Martynkien, J. Olszewski, P. Mergo, M. Makara, K. Poturaj, and W. Urbańczyk, "Spectral-domain measurement of birefringence and characteristics of a side-hole microstructured fiber," Sensors, vol. 13, pp. 11424-11438, 2013.

[5] Zh. Ding, Ch. Wang, K. Liu, Y. Liu, G. Xu, J. Jiang, Y. Guo, and T. Liu, "Distributed measurements of external force induced local birefringence in spun highly birefringent optical fibers using polarimetric OFDR," Optics Express, vol. 27, no.2, pp. 951 - 964, 2019

[6] L. Yuan, G. Zhang, and Q. Li, "Interaction model for a Hi-Bi fiber optic ultrasonic sensor and the host material," Journal of Intelligent Material Systems and Structures, vol. 18, pp. 875-878, 2007.
[7] A. Layeghi and H. Latifi, "Magnetic field vector sensor by a nonadiabatic tapered $\mathrm{Hi}-\mathrm{Bi}$ fiber and ferrofluid nanoparticles," Optics and Laser Technology, vol. 102, pp. 184-190, 2018.

[8] O. Frazão, J. M. Baptista, and J. L. Santos, "Recent advances in highbirefringence fiber loop mirror sensors," Sensors, vol. 7, pp. 29702983, 2007.

[9] G. Keiser, Optical Fiber Communications, 3rd ed., McGraw-Hill, Singapore, 2000.

[10] E. De la Rosa, L. A. Zenteno, A. N. Starodumov, and D. Monzon, "All-fiber absolute temperature sensor using an unbalanced highbirefringence Sagnac loop," Optics Letters, vol. 22, no. 7, pp. 481483, 1997.

[11] Y. Liu, B. Liu, X. Feng, W. Zhang, G. Zhou, Sh. Yuan, G. Kai, and $\mathrm{X}$. Dong, "High-birefringence fiber loop mirrors and their applications as sensors," Applied Optics, vol. 44, no. 12, pp. 23822390, 2005

[12] M. Born, and E. Wolf, Principles of Optics (Cambridge University Press, Cambridge - 2003).

[13] N. Tarjányi, and D. Káčik, "Birefringence of pure and doped lithium niobate crystals,” Proc. SPIE 10976, 1097616 (2018). 